\title{
USING MOBILE TECHNOLOGY TO CROWDSOURCE THE AUGMENTATION OF DEEP LEARNING DATASETS
}

\author{
Chantelle Saliba and Dylan Seychell \\ Department of Artificial Intelligence, University of Malta \\ Msida MSD2080, Malta
}

\begin{abstract}
In the past decade, mobile communications have seen drastic changes and improvements with an estimate of over 3.5 billion mobile phone users worldwide. In addition, the average mobile phone has gone from being a simple communication device to a smartphone device capable of web browsing, video conferencing, gaming, photography, and videography and intelligent applications. For this reason, companies and industries have been exploring this technology to create opportunities to enhance their communications with clients and to create further business opportunities. In this research, we analyze the approach of using mobile technologies as a technique to crowdsource data that would be used to enhance research by creating digital resources. In today's modern and technological world there are areas and fields which are still unexplored by technology due to their lack of digital resources. Modern machine learning techniques such as deep learning methods, require a large volume of data that is not always available. Such a case is the example of classifying Maltese flora. Malta is a small island in the middle of the Mediterranean with an area of $316 \mathrm{~km}^{2}$. Being such a small island with unique and indigenous flora makes it a challenging feat to find already available digital data to be able to conduct technological research. For this reason, we turn to mobile technology and how this can aid in the collection of such data to augment existing datasets that enhance academic research and render classification more effective and feasible.
\end{abstract}

\section{KEYWORDS}

Mobile Technology, Research, Data, Crowdsourcing, Machine Learning

\section{INTRODUCTION}

Mobile technology has seen great advancements in the past years and mobile technology has become more and more advanced, encouraging the population of mobile users to drastically infatuate worldwide. Smartphones nowadays are equipped with higher memory storages and higher performance ratings. In addition, the simple mobile phone is now capable of web browsing, photography, videography and gaming. Organizations and business have taken this opportunity to create mobile applications to feel closer to their customers and audience and to understand better the customer needs and wants. In this research, we would be analyzing the prospects of using mobile communications for academic research. Academic research has been strengthened by technological advancement, helping in the collection of data and the analysis of data by performing complex computations that apply machine learning techniques to analyze it accurately and efficiently. In this research, we will be analyzing the concept of implementing a mobile communication medium to crowdsource data to create digital resources that will be used to enhance deep learning datasets that enable better analysis of artefacts. For technology to aid academic research, digital resources are required and even though we live in an advanced and technological era, there are unexplored fields by technology due to their lack of digital resources. In this research, we would be analyzing Maltese flora [Lanfranco and Lanfranco, 2003] and the idea of introducing an image classification system using deep learning algorithms from the digital data crowdsourced by a mobile technology system.

\subsection{Motivation}

The motivation behind this system has two different perspectives. Firstly, the system revolves around the idea of collecting data that is sparse and needed to enhance datasets for deep learning algorithms. Gathering 
this digital data will enable further research as well as introduce other technological techniques such as machine learning to unexplored fields.

In addition, by conducting this experiment on an island's botany, we would be shifting the mobile user's attention to areas that are usually taken for granted. Moreover, through this study we will be raising awareness about Malta's flora and natural surroundings and its conservation. This is further enhanced by other data, such as location, available on mobile phones that adds context and features to the visual data being collected.

\section{BACKGROUND RESEARCH}

\subsection{Mobile Technology}

Mobile phones and smartphones have seen drastic changes and improvement in both performance, and features. The simple mobile phone that used to enable communication between two distant individuals, has now been transformed into a technological device capable of web browsing, video conferencing, videography, phone photography and gaming. Smartphones nowadays are also equipped with high-performance processors and higher memory storage. This encouraged the creation of applications from various fields and areas with personal or global interest to communicate with mobile users. By the year 2013, there were roughly 13000 applications that provided medical and health advice [Trueland, 2013]. Business and retailers have also taken this opportunity to expand their horizons and use this medium as an excellent business and marketing tool. Businesses have been making use of applications to enhance customer experience [Mclean et al, 2018] and as a branded marketing strategy [Li, 2018] where the traditional membership card is being traded to a more attractive and gamified mobile application. A substantial number of applications are also for educational purposes both for formal learning and also for informal learning [Churchill et al, 2016]. Demographically the largest number of mobile users is between the ages of 18 and 29 , which makes it a perfect opportunity for educational institutions to provide learning mechanisms beyond the traditional classroom [Crompton and Burke, 2018]. Incorporating mobile learning to the classrooms has proven to improve the student's motivation and engagement towards their education [Camilleri et al, 2019].

\subsubsection{Mobile Communication for Data Collection}

With the population of mobile phone users increasing globally, mobile communication could be considered as an excellent source of data collection. Data collection can be performed for various institutions from businesses to understand their customer's preferences and trends to academics that gather data for further research. The expansion of mobile phones has enabled data collection to be made more efficient and representative by reaching a more geographically spread number of people. Conducting survey research by making use of mobile technology will also enable the researchers to make use of the location of the individuals participating in the survey by making use of GPS features. This will enable the researchers to have more in-depth information in understanding the participant. Furthermore, nowadays smartphones are equipped with cameras and audio recording features that can enhance data value as well as provide new opportunities for data collection. Moreover, gathering information through technological means gives the advantage of real-time data transfer and the information gathered can be manipulated and analyzed in real-time, therefore, saving costs while also being environmentally friendly. Data collection via a mobile communication system can be conducted through online surveys, questionnaires and mobile applications [Barzilay, 2019]. When conducting data collection via mobile communication, the privacy of the participants should be safeguarded and all the necessary precautions should be taken. The researchers should ensure that all the information is protected. GPS tracking and sharing of personal information and visual imagery should be done only upon informing the participant and getting the necessary permissions [Link et al, 2014].

\subsubsection{User Engagement through Mobile Communication}

The population of mobile users is increasing because mobile features are becoming more advanced and applications are becoming more engaging and addictive especially amongst college students [Kumar and Gera, 2016]. The methods and techniques that stimulate motivation depend on the area to which the application is being used. A learning application can be made motivating by taking into consideration the user's background as well as information about the environment before generating content. [Dingli and Seychell, 2012] generated a more engaging learning application by creating a system that makes use of 
3 layers where the innermost layer represents the information generated from the user's environment and the outer layer represents the information that is most related to the user's profile. A technique that is widely used in learning applications as well as in business applications is gamification. Gamification is the process of adopting game design mechanics to mobile applications [Cechetti et al, 2017]. This engages the user through a number of game elements such as rewards, points, characters and personalized feedback [Tóth and Tóvölgyi, 2016].

\subsection{Machine Learning}

Machine Learning (ML) is one of the fundamental concepts of Artificial Intelligence and it is playing a key role in the advancement of technology. One can find ML in various fields such as in robotics, computer games, data mining, medical diagnosis, social media services, pattern recognition, natural language processing, product recommendation, online fraud detection to name a few [Ray, 2019]. Conventional machine learning techniques made use of extensive domain expertise that was transformed to data that the machine learning classifier was able to interpret and classify. Deep Learning algorithms are the new generation of machine learning techniques which are composed of multiple processing layers that will learn the raw data through a number of steps to which in each step the algorithm would be discovering more and more about the data. Deep learning has proven to outperform traditional machine learning algorithms in various fields such image recognition and classification. This research addresses the challenge that such techniques face when handling smaller sets of data and we propose a method that uses mobile technology to enhance the datasets that are needed for such techniques.

\subsubsection{Convolutional Neural Networks}

A Convolutional Neural Network (CNN) is a deep learning algorithm that is said to be biologically inspired [Ray, 2019]. The Convolutional Neural Network's architecture is composed of a number of stages. It first starts by receiving the data and passes it through a series of convolutional layers and pooling layers. The convolutional layer is used to learn the features by computing convolutions between the input and a set of kernels or filters. A kernel or filter can perform operations such as edge detection and sharpening. A non-linear activation function such as the rectified linear unit (ReLU) function is then performed on the convolutions. This introduces non-linearity in the convolutional network. The pooling layer tries to reduce the number of parameters by merging similar features while retaining important information [Lecun et al., 2015]. After feature extraction, the classification component receives the data as a matrix and after performing flatting, passes it through one or more fully connected layers. The results will then be passed through an activation function such as softmax or sigmoid to classify the outputs. CNNs can be seen at work in various areas such as music [Vishnupriya and Meenakshi, 2018] and hand gesture recognition [Han et al., 2016].

\subsubsection{Convolutional Neural Networks for Flower Classification}

Given their success rate in image classification, [Liu et al., 2016] suggested an eight layer CNN to classify flower images. The first five layers were convolutional layers while the remaining three were fully connected layers. This system was able to distinguish between 79 different flower classes and achieved an accuracy rate of $76.54 \%$. Techniques that were introduced in the neural network to generate a higher accuracy rate included local response normalization, overlapping pooling and dropout to prevent saturation and overfitting in both the pooling layers and fully-connected layers.

\subsection{Maltese Flora}

Malta is composed of a group of small islands with the main islands being Malta, Gozo and Comino with Malta being the mainland. All the islands compose an average of $316 \mathrm{~km}^{2}$. In Malta, due to our central position in the Mediterranean Sea, one finds flora that is located in southern Europe and northern Africa as well as flora that is indigenous to the Maltese islands [Lanfranco, 2007]. 


\section{METHODOLOGY}

The introduction of a mobile communication system that will crowdsource data is human-centric and therefore it requires human input and collaboration. Hence, the methodology of this system was divided into two phases. A feasibility study was firstly conducted by performing an online survey about the people's opinion about crowdsourcing data. In this study we analyzed the participant's profiles and their relation with the Maltese nature and if they frequently go for hikes or picnics in the Maltese countryside. Moreover, in this survey, it was asked about their willingness to collaborate in this study by submitting their photos of flowers and their opinion about a mobile application about the Maltese flora. The second phase of the methodology included the designing of a proposed solution while keeping the data and the conclusions that were derived from the research in mind.

\subsection{Conducted Research}

In this research, a survey was conducted about the people's opinion of crowdsourcing data. In this survey, we also analyzed people's mobile behavioral patterns. In this research, a total of 243 participants took place where all the participants are of either Maltese descendent or British descendent between the ages of 12 and 80. All the participants that took place resided in one of the Maltese islands.

\subsubsection{User's Profile}

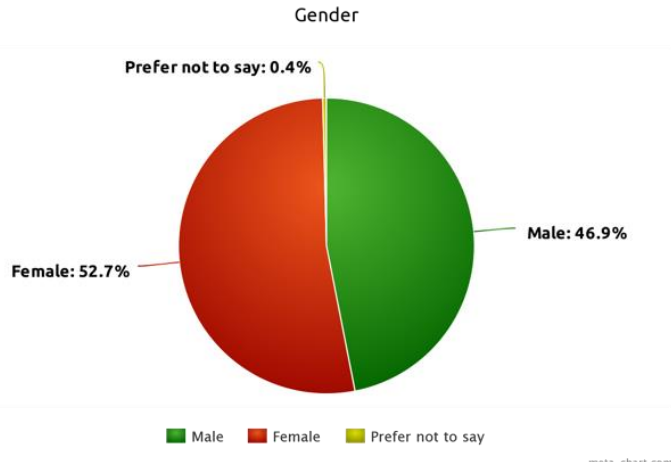

Figure 1. Gender of Participants

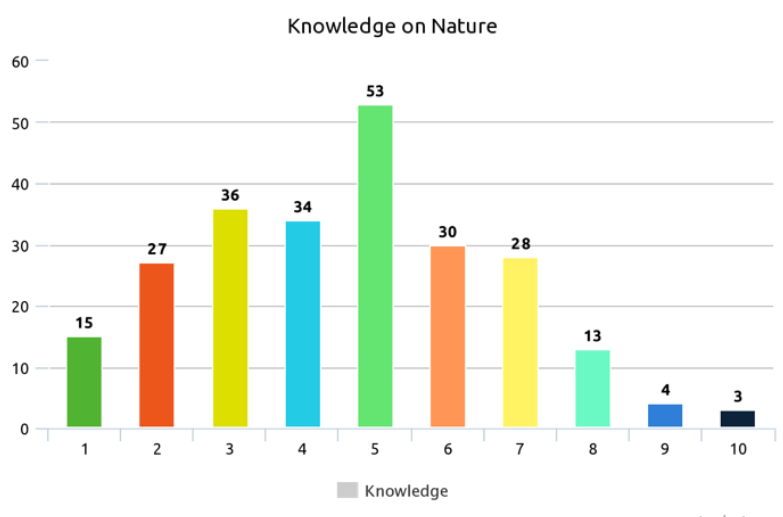

Figure 2. Knowledge on Nature

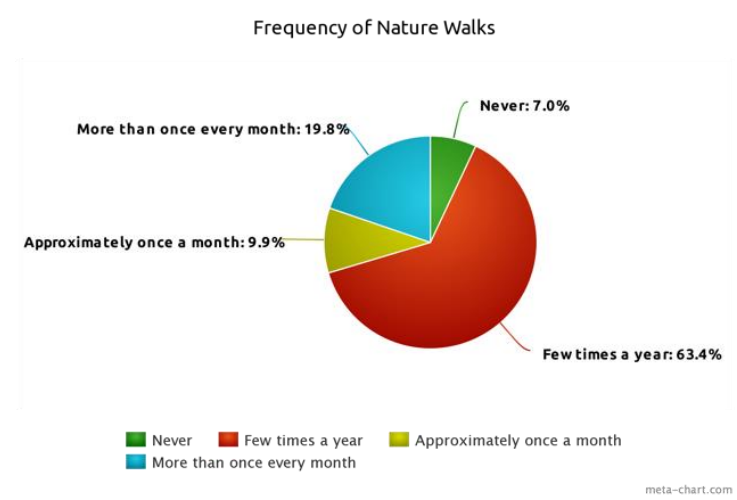

Figure 3. Frequency of Nature Walks

In this research, the male and female ratio was almost balanced with a $52.7 \%$ female representation and a $46.9 \%$ male representation as shown in Figure $1.0 .4 \%$ of the population preferred not to affiliate with any gender. 
The research being conducted is human dependent and therefore understanding the participant needs and their willingness to contribute is important. Their contribution is also dependable on the area to which the academic research is being conducted. Therefore, a part of the conducted research involved around the user's opinion about Maltese nature and how knowledgeable the residents feel about the Maltese flora. It was concluded that the Maltese do not feel as knowledgeable as can be shown in Figure 2 were $21.8 \%$ of the participants ranked their knowledge as a 5 which is the middle of the scale. $32.1 \%$ of all the participants gave a scoring rate of higher than 5 ; while $46.1 \%$ gave a scoring of lower than 5 . This show that overall the participants gave a scoring of 5 or lower. When asked about how frequent the participants go for walks or hikes in nature, the results showed that the Maltese with a percentage of $63.4 \%$ go for a walk a few times a year. In addition, there were $19.8 \%$ that stated that they go for hikes more than once every month, as shown in Figure 3. This research also considered the main reasons why the participants go into nature and some of the most popular opinions included admiring nature $(74.9 \%)$, relaxing $(74.1 \%)$, and taking photos $(53.1 \%)$. Other activities included hanging out with family and friends and performing physical activities such as running and jogging.

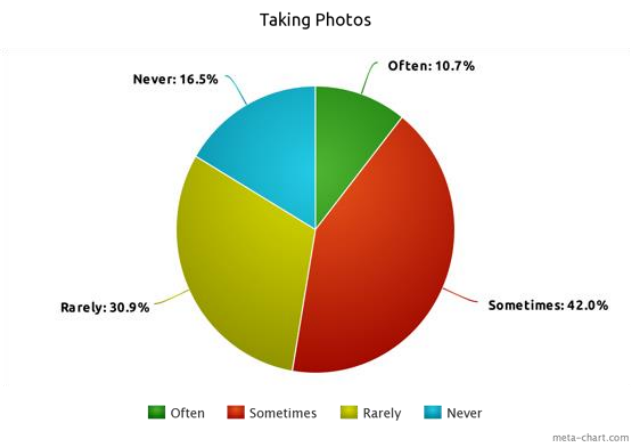

Figure 4. Photographing Photos

To gather digital resources about Maltese flora, the participants will need to photograph flowers. This research asked the participants how often they take photos of flowers. It results that the majority sometimes take flowers with a percentage of $42 \%$ as shown in Figure 4.

\subsubsection{Mobile Behavior Patterns}

An important element when it comes to data crowdsourcing is to understand the participants' mobile behaviour patterns and engagement. Gathering data using such methods involves that the audience is familiar with the technological medium and makes use of smartphone features such as the mobiles cameras and application. The participants in this study made use of two of the most famous operating systems: android and $\mathrm{iOS}$ with $83.5 \%$ of the participants making use of an android operating system and $16.5 \%$ of the participants making use of iOS as can be shown in Figure 5.

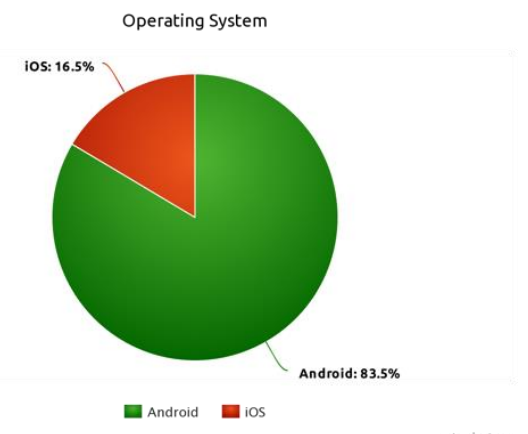

Figure 5. Operating Systems

It was found that the majority make use of Internet browsing (92.2\%), Social Media (90.5\%) and the camera $(89.3 \%)$ when it comes to smartphone features. Another widely used feature is text messaging. 
In addition, it was concluded that $91.8 \%$ have applications installed on their smartphone. Only $2.5 \%$ stated that they do not have applications on their phone while $5.8 \%$ stated that they are not sure if they have applications or not, as shown in Figure 6. This result shows that an application for crowdsourcing might be a possible solution since the number of application users is high.

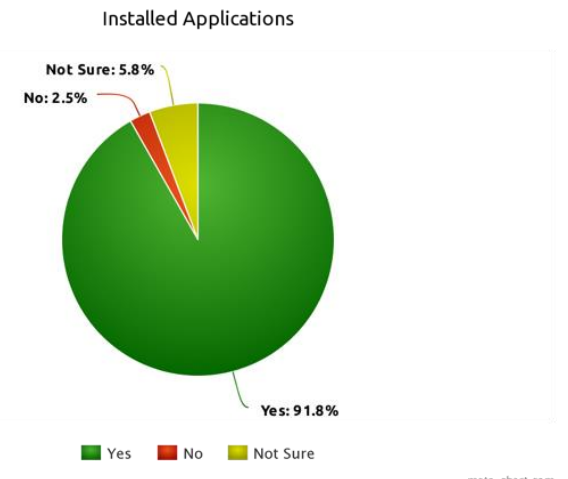

Figure 6. Installed Applications

\subsubsection{Data Crowdsourcing}

In its core principle crowdsourcing has the willingness of the participants to form part of the research and include themselves in the data gathering process. This can be achieved if the participants are willing to participate in the research and provide the data they collected. The study shows that $51.4 \%$ of the people that participated would be willing to share their photos of flowers. On the other hand, $15.2 \%$ would not be willing to share their photos even for research purposes. The other $33.3 \%$ said that it depends on the situation and the case study.

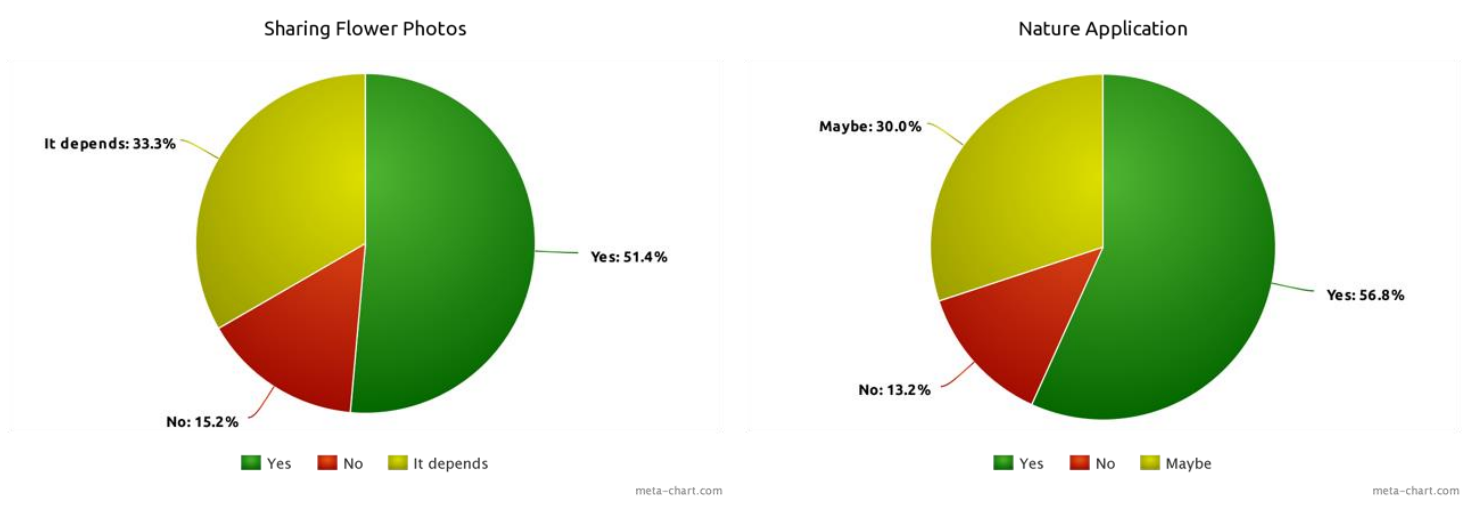

Figure 7. Willingness to share Flower Photos

Figure 8. Use of a Nature related Application

A mobile application will be a good technique to enable crowdsourcing of data. As shown in Figure 8, the majority of the participants said that they would be interested in an application that is related to nature and that would provide related information. $30 \%$ answered that they are not sure if they would make use of such an application while $13.2 \%$ said that would not make use of such an application.

\subsection{Proposed Solution}

From the research conducted, it could be concluded that an application would be an ideal medium to crowdsource data. The application could provide the users with information about the species of the photographed image by making use of machine learning techniques such as a convolutional neural network (CNN) while storing the image photographed. 


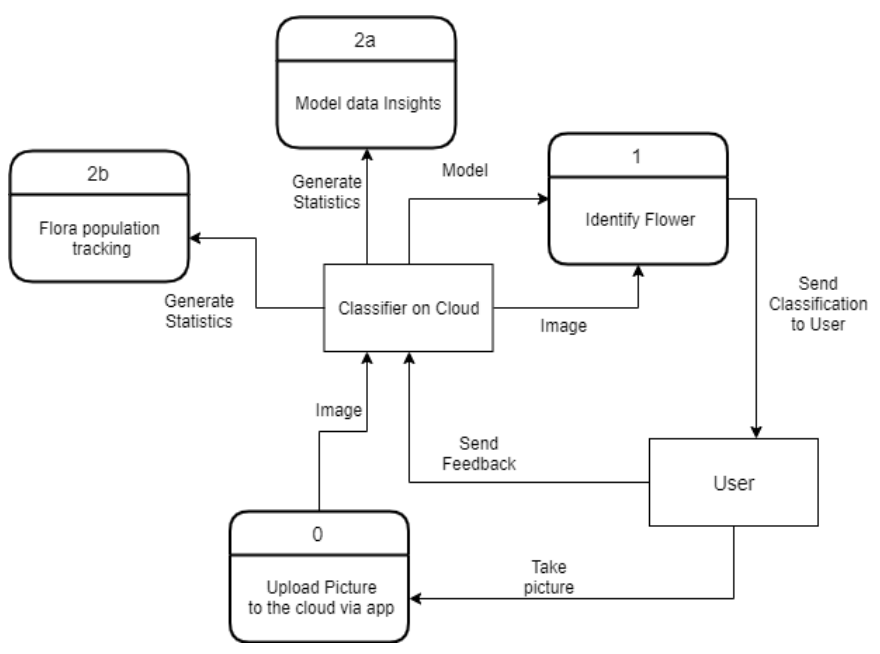

Figure 9. Block Diagram of Proposed Solution

The block diagram of the proposed solution can be seen in Figure 9. The participant will be interacting with the system through the use of an application and upon taking a photo through the application; the system will send this image to the cloud. This image will be stored in the cloud and will be used to add to the current digital resources related to Maltese Flora. A machine learning model would also be located in the cloud and this model will return the label along with some images of the same determined label. In addition, the app user will be requested to provide feedback on the label and the related images returned. This feedback would be used to generate insights on the machine learning model.

A prototype application has been set up for this purpose as shown in Figure 10. The first image in this figure shows the first part of the application where the user will be able to upload a photo of a flower to the flower either by choosing an image from the gallery or by taking a photo from the camera. This photo will be sent to the cloud and apart from storing this image to store as digital resources, the system will make use of a model to classify that photo with a level of confidence. The second image of the Figure shows the second part of the application where the user will be shown similar images and the user will be able to give feedback to the model by submitting the rating stars. The application is making use of Google services such as Firebase $^{1}$ and the ML Kit ${ }^{2}$. The application is currently locally based and can classify between five different types of flowers: roses, sunflowers, dandelions, tulips and daisies. This will, however, be extended and fine-tuned to Maltese flora to enable further testing and implementation.
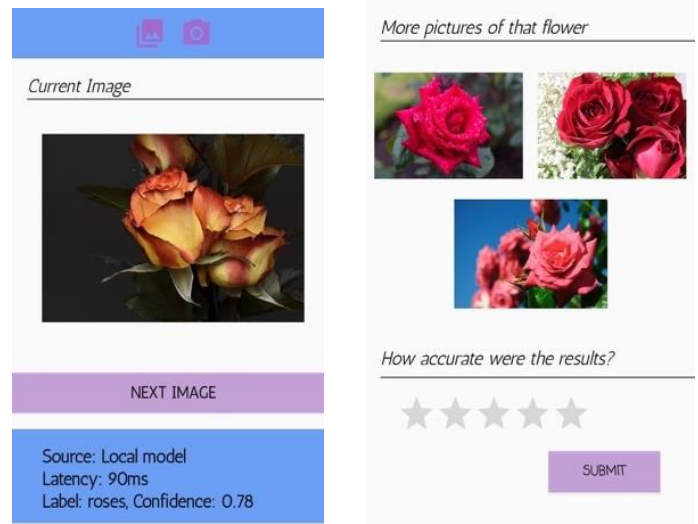

Figure 10. Prototype Application

\footnotetext{
${ }^{1}$ Google's cloud mobile back-end platform, available at https://firebase.google.com/

${ }^{2}$ Machine Learning for mobile development by Google, available at https://firebase.google.com/products/ml-kit
} 


\section{CONCLUSION}

In this research study, presented the concept of creating a mobile application that uses crowdsourcing data to improve a dataset for machine learning models. The digital data collected will then be used for academic research and for the implementation of image classification through the use of machine learning techniques to classify Maltese flora that as of currently there is limited digital data available. This system would enable digital data to be collected from the citizens residing or visiting Malta through the use of an application with the mobile's photography features while providing them with information about the photographed flower. A survey was conducted to analyze the opinion of the citizens, about data crowdsourcing and it was concluded that only $13.2 \%$ stated that they will not make use of such an application, while $15.2 \%$ stated that they will not be willing to share their photos of flowers for academic research. This system can be applied to other areas and fields that have limited digital resources that would enable the collection of data for further academic research.

\section{REFERENCES}

Barzilay, M., 2019. Data Collection and Mobile Technologies. 10.13140/RG.2.2.31541.93929.

Camilleri, A., et al, 2019. Mobile learning via educational apps: an interpretative study.

Cechetti, N. P. et al., 2017. Gamification strategies for mobile device applications: A systematic review. 12th Iberian Conference on Information Systems and Technologies (CISTI). Lisbon, Portugal, pp. 1-7.

Churchill et al., 2016. Mobile Learning Design Theories and Application 1st ed. 2016.

Crompton, H. \& Burke, D., 2018. The use of mobile learning in higher education: A systematic review. Computers \& Education, 123, pp.53-64.

Dingli, A. and Seychell, D., 2012. Motivating learning through mobile interaction. Proceedings of IADIS International Conference on Mobile Learning. Berlin, Germany, pp. 271-274.

Fox, B.I. \& Felkey, B.G., 2014. Quick Survey of Smartphone Features and Functions. Hospital Pharmacy, 49(6), pp.585-586.

Han, M., et al., 2016. Visual hand gesture recognition with convolution neural network. 2016 17th IEEE/ACIS International Conference on Software Engineering, Artificial Intelligence, Networking and Parallel/Distributed Computing (SNPD). Shanghai, pp. 287-291.

Kumar, A. \& Gera, N., 2016. Mobile Addiction and Mental Health of College Students. Educational Quest, 7(2), pp. 87-90.

Lanfranco, E. and Lanfranco, G., 2003. Il-Flora Maltija. Pubblikazzjonijiet Indipendenza, Malta.

Lanfranco, G., 2007. Fjuri Slavag. Wise Old Publications, Malta.

Lecun, Y., et al., 2015. Deep learning. Nature, 521(7553), pp.436-444.

Li, C.-Y., 2018. Consumer behavior in switching between membership cards and mobile applications: The case of Starbucks. Computers in Human Behavior, 84, pp.171-184.

Link, M.W. et al., 2014. Mobile Technologies for Conducting, Augmenting and Potentially Replacing Surveys. Public Opinion Quarterly, 78(4), pp.779-787.

Liu, Y., et al., 2016. Flower classification via convolutional neural network. 2016 IEEE International Conference on Functional-Structural Plant Growth Modeling, Simulation, Visualization and Applications (FSPMA), Qingdao, pp. 110-116.

Mclean, G. et al, 2018. Developing a Mobile Applications Customer Experience Model (MACE)- Implications for Retailers. Journal of Business Research, 85, pp.325-336.

Ray. S., 2019. A Quick Review of Machine Learning Algorithms. 2019 International Conference on Machine Learning, Big Data, Cloud and Parallel Computing (COMITCon). Faridabad, India, pp. 35-39.

Tóth, Á. \& Tóvölgyi, S., 2016. The introduction of gamification: A review paper about the applied gamification in the smartphone applications. 000213-000218. 10.1109/CogInfoCom.2016.7804551.

Trueland, J., 2013. A smart use for mobiles. Nursing Standard (through 2013), 27(36), pp.16-8.

Vishnupriya, S., and Meenakshi, K., 2018. Automatic Music Genre Classification using Convolution Neural Network. 2018 International Conference on Computer Communication and Informatics (ICCCI). Coimbatore, pp. 1-4. 